\title{
SIMPLE PROOFS OF NOWHERE-DIFFERENTIABILITY FOR WEIERSTRASS'S FUNCTION AND CASES OF SLOW GROWTH
}

\author{
JON JOHNSEN
}

\begin{abstract}
Using a few basics from integration theory, a short proof of nowhere-differentiability of Weierstrass functions is given. Restated in terms of the Fourier transformation, the method consists in principle of a second microlocalisation, which is used to derive two general results on existence of nowhere differentiable functions. Examples are given in which the frequencies are of polynomial growth and of almost quadratic growth as a borderline case.
\end{abstract}

\section{INTRODUCTION}

In 1872, K. Weierstrass presented his famous example of a nowhere differentiable function $W$ on the real line $\mathbb{R}$. With two real parameters $b \geq a>1$, this may be written as

$$
W(t)=\sum_{j=0}^{\infty} a^{-j} \cos \left(b^{j} t\right), \quad t \in \mathbb{R} .
$$

Weierstrass proved that $W$ is continuous at every $t_{0} \in \mathbb{R}$, but not differentiable at any $t_{0} \in \mathbb{R}$ if

$$
\frac{b}{a}>1+\frac{3 \pi}{2}, \quad b \text { is an odd integer. }
$$

Subsequently several mathematicians attempted to relax condition (1.2), but with limited luck. Much later G. H. Hardy [Har16] was able to remove it:

Theorem 1.1 (Hardy 1916). For every real number $b \geq a>1$ the functions

$$
W(t)=\sum_{j=0}^{\infty} a^{-j} \cos \left(b^{j} t\right), \quad S(t)=\sum_{j=0}^{\infty} a^{-j} \sin \left(b^{j} t\right),
$$

are bounded and continuous on $\mathbb{R}$, but have no points of differentiability.

The assumption $b \geq a$ here is optimal for every $a>1$, for $W$ is in $C^{1}(\mathbb{R})$ whenever $\frac{b}{a}<1$, due to uniform convergence of the derivatives. (Strangely this was unobserved in [Har16, Sect. 1.2], where Hardy sought to justify the sufficient condition $b \geq a$ as being more natural than eg (1.2).) Hardy also proved that $S^{\prime}(0)=+\infty$ for

$$
1<a \leq b<2 a-1
$$

2000 Mathematics Subject Classification. 26A27.

Key words and phrases. nowhere-differentiability, Weierstrass function, lacunary Fourier series, second microlocalisation

Appeared in J. Fourier Anal. Appl. 16 (2010), pp. 17--33. 
so then the graph of $S(t)$ is not rough at $t=0\left(\right.$ similarly $W^{\prime}(\pi / 2)=+\infty$ if in addition $\left.b \in 4 \mathbb{N}+1\right)$. However, Hardy's treatment is not entirely elementary and yet it fills ca. 15 pages.

It is perhaps partly for this reason that attempts have been made over the years to find other examples. These have often involved a replacement of the sine and cosine above by a function with a zig-zag graph, the first one due to T. Takagi [Tak03] who introduced $t \mapsto \sum_{j=0}^{\infty} 2^{-j} \operatorname{dist}\left(2^{j} t, \mathbb{Z}\right)$.

However, the price is that the partial sums are not $C^{1}$ for such functions, and due to the dilations every $x \in \mathbb{R}$ is a limit $x=\lim r_{N}$ where each $r_{N} \in \mathbb{Q}$ is a point at which the $N^{\text {th }}$ partial sum has no derivatives; whence nowhere-differentiability of the sum function is less startling. Nevertheless, a fine example of this sort was given in just 13 lines by J. McCarthy [McC53].

Somewhat surprisingly, there is an equally short proof of nowhere-differentiability for $W$ and $S$, using a few basics of integration theory. This is explained below in the introduction.

It is a major purpose of this paper to show that the simple method has an easy extension to large classes of nowhere differentiable functions. Thus the main part of the paper contains two general theorems, of which at least the last should be a novelty, and it ends with new examples with slow increase of the frequencies.

Remark 1.2. By a well-known reasoning, $W$ is nowhere-differentiable since the $j^{\text {th }}$ term cannot cancel the oscillations of the previous ones: it is out of phase with previous terms as $b>1$ and the amplitudes decay exponentially since $\frac{1}{a}<1$; as $b \geq a>1$ the combined effect is large enough (vindicated by the optimality of $b \geq a$ noted after Theorem 1.1). However, it will be shown in Section 4 that frequencies growing almost quadratically suffice for nowhere-differentiability.

To present the ideas in a clearer way, one may consider the following function $f_{\theta}$ which (in this paper) serves as a typical nowhere differentiable function,

$$
f_{\theta}(t)=\sum_{j=0}^{\infty} 2^{-j \theta} e^{\mathrm{i} 2^{j} t}, \quad 0<\theta \leq 1
$$

It is convenient to choose an auxiliary function $\chi: \mathbb{R} \rightarrow \mathbb{C}$ thus: the Fourier transformed function $\mathscr{F} \chi(\tau)=\hat{\chi}(\tau)=\int_{\mathbb{R}} e^{-\mathrm{i} t \tau} \chi(t) d t$ is chosen as a $C^{\infty}$-function fulfilling

$$
\hat{\chi}(1)=1, \quad \hat{\chi}(\tau)=0 \text { for } \tau \notin] \frac{1}{2}, 2[;
$$

for example by setting $\hat{\chi}(\tau)=\exp \left(2-\frac{1}{(2-\tau)(\tau-1 / 2)}\right)$ for $\left.\tau \in\right] \frac{1}{2}, 2[$.

Using (1.6) it is easy to show that $\chi(t)=\mathscr{F}^{-1} \hat{\chi}(t)=\frac{1}{2 \pi} \int_{\mathbb{R}} e^{\mathrm{i} t \tau} \hat{\chi}(\tau) d \tau$ is continuous and that for each $k \in \mathbb{N}_{0}$ the function $t^{k} \chi(t)=\mathscr{F}^{-1}\left(\mathrm{i}^{k} \hat{\chi}^{(k)}\right)$ is bounded (by $\left.\sup \left|\hat{\chi}^{(k)}\right|\right)$. Therefore $\chi$ is integrable, ie $\chi \in L_{1}(\mathbb{R})$, and clearly $\int \chi d t=\hat{\chi}(0)=0$. 
With this preparation, the function $f_{\theta}$ is particularly simple to treat, using only ordinary exercises in integration theory: First one may introduce the convolution

$$
2^{k} \chi\left(2^{k} \cdot\right) * f_{\theta}\left(t_{0}\right)=\int_{\mathbb{R}} 2^{k} \chi\left(2^{k} t\right) f_{\theta}\left(t_{0}-t\right) d t
$$

which is in $L_{\infty}(\mathbb{R})$ since $f_{\theta} \in L_{\infty}(\mathbb{R})$ and $\chi \in L_{1}(\mathbb{R})$. Secondly this will be analysed in two different ways in the proof of

Proposition 1.3. For $0<\theta \leq 1$ the function $f_{\theta}(t)=\sum_{j=0}^{\infty} 2^{-j \theta} e^{\mathrm{i} 2^{j} t}$ is a continuous $2 \pi$-periodic, hence bounded function $f_{\theta}: \mathbb{R} \rightarrow \mathbb{C}$ without points of differentiability.

Proof. By uniform convergence $f_{\theta}$ is for $\theta>0$ a continuous $2 \pi$-periodic and bounded function; this follows from Weierstrass's majorant criterion as $\sum 2^{-j \theta}<\infty$.

Inserting the series defining $f_{\theta}$ into (1.7), Lebesgue's theorem on majorised convergence allows the sum and integral to be interchanged (eg with $\frac{2^{k}}{1-2^{-\theta}}\left|\chi\left(2^{k} t\right)\right|$ as a majorant), ie

$$
\begin{aligned}
2^{k} \chi\left(2^{k} \cdot\right) * f_{\theta}\left(t_{0}\right) & =\lim _{N \rightarrow \infty} \sum_{j=0}^{N} 2^{-j \theta} \int_{\mathbb{R}} 2^{k} \chi\left(2^{k} t\right) e^{\mathrm{i} 2^{j}\left(t_{0}-t\right)} d t \\
& =\sum_{j=0}^{\infty} 2^{-j \theta} e^{\mathrm{i} 2^{j} t_{0}} \int_{\mathbb{R}} e^{-\mathrm{i} z 2^{j-k}} \chi(z) d z=2^{-k \theta} e^{\mathrm{i} 2^{k} t_{0}} \hat{\chi}(1)=2^{-k \theta} e^{\mathrm{i} 2^{k} t_{0}} .
\end{aligned}
$$

Here it was also used that $\hat{\chi}\left(2^{j-k}\right)=1$ for $j=k$ and equals 0 for $j \neq k$.

Moreover, since $f_{\theta}\left(t_{0}\right) \int_{\mathbb{R}} \chi d z=0$ (cf the note prior to the proposition) this gives

$$
2^{-k \theta} e^{i 2^{k} t_{0}}=2^{k} \chi\left(2^{k} \cdot\right) * f_{\theta}\left(t_{0}\right)=\int_{\mathbb{R}} \chi(z)\left(f_{\theta}\left(t_{0}-2^{-k} z\right)-f_{\theta}\left(t_{0}\right)\right) d z .
$$

So if $f_{\theta}$ were differentiable at $t_{0}, F(h):=\frac{1}{h}\left(f_{\theta}\left(t_{0}+h\right)-f_{\theta}\left(t_{0}\right)\right)$ would define a function in $C(\mathbb{R}) \cap L_{\infty}(\mathbb{R})$ for which $F(0)=f^{\prime}\left(t_{0}\right)$, and Lebesgue's theorem, applied with $|z \chi(z)| \sup _{\mathbb{R}}|F|$ as the majorant, would imply that

$$
-2^{(1-\theta) k} e^{\mathrm{i} 2^{k} t_{0}}=\int F\left(-2^{-k} z\right) z \chi(z) d z \underset{k \rightarrow \infty}{\longrightarrow} f^{\prime}\left(t_{0}\right) \int_{\mathbb{R}} z \chi(z) d z=f^{\prime}\left(t_{0}\right) \mathrm{i} \frac{d \hat{\chi}}{d \tau}(0)=0
$$

hence that $1-\theta<0$. This would contradict the assumption that $\theta \leq 1$.

By now this argument is of course of a classical nature, although not well established in the literature. Eg, recently R. Shakarchi and E. M. Stein treated nowhere-differentiability of $f_{\theta}$ in Thm. 3.1 of Chap. 1 in their treatise [SS03] with a method they described thus: "The proof of the theorem is really the story of three methods of summing a Fourier series... partial sums... Cesaro summability... delayed means." However, they covered $0<\theta<1$ in a few pages with refinements for $\theta=1$ sketched there in Problem 5.8 based on the Poisson summation formula.

The present proofs are not confined to periodic functions (cf the next section), for the theory of lacunary Fourier series is replaced by the Fourier transformation $\mathscr{F}$ and its basic properties. 
Moreover, also Hardy's theorem can be obtained in this way, with a few modifications. The main point is to keep the factor $e^{\mathrm{i} 2^{k} t_{0}}$ instead of introducing $\cos \left(2^{k} t_{0}\right)$ and $\sin \left(2^{k} t_{0}\right)$ that appear in $W$ and $S$, but do not a priori stay away from 0 as $k \rightarrow \infty$. Luckily this difficulty (which was dealt with at length in [Har16]) disappears with the present approach:

Proof of Theorem 1.1 As $a>1$, clearly $W \in C(\mathbb{R}) \cap L_{\infty}$. Since $b>1$ it may in this proof be arranged that $\hat{\chi}(1)=1$ and $\hat{\chi}(\tau) \neq 0$ only for $\frac{1}{b}<\tau<b$. As for $f_{\theta}$ this gives, by Euler's formula,

$$
b^{k} \chi\left(b^{k} \cdot\right) * W\left(t_{0}\right)=\sum_{j=0}^{\infty} a^{-j} \int_{\mathbb{R}} b^{k} \chi\left(b^{k} t\right) \frac{1}{2}\left(e^{\mathrm{i} b^{j}\left(t_{0}-t\right)}+e^{\mathrm{i} b^{j}\left(t-t_{0}\right)}\right) d t .
$$

The term $e^{\mathrm{i} b^{j}\left(t-t_{0}\right)}$ is redundant here, for $z:=t b^{k}$ yields $\int e^{\mathrm{i} b^{j} t} \chi\left(b^{k} t\right) b^{k} d t=\int e^{\mathrm{i} z b^{j-k}} \chi(z) d z=$ $\hat{\chi}\left(-b^{j-k}\right)=0$, as $\hat{\chi}$ vanishes on $\left.]-\infty, 0\right]$. So as in (1.8), one has $b^{k} \chi\left(b^{k} \cdot\right) * W\left(t_{0}\right)=\frac{e^{i b^{k} t_{0}}}{2 a^{k}}$.

Hence existence of $W^{\prime}\left(t_{0}\right)$ would imply that $\lim _{k}\left(\frac{b}{a}\right)^{k} e^{i b^{k} t_{0}}=0$; cf (1.9)-(1.10). This would contradict that $b \geq a$, so $W$ is nowhere differentiable. Similarly $S(t)$ is so.

It is known that nowhere-differentiability of $W$ can be derived with wavelets, cf [ [Hol95]; an elementary explanation has been given in [BD92], but only for $b>a$. In comparison the above proofs are short and cover all cases through "first principles" of integration theory.

In Section 2 a general result on nowhere differentiable functions is given. Refining a dilation argument, a further extension is found in Section 3, including functions with polynomial frequency growth. Borderline cases with quasi-quadratic growth are given in Section 4.

Remark 1.4. In the proof of Theorem 1.1, Lebesgue's theorem on majorised convergence is the most advanced part. As this result appeared in 1908, cf [Leb08, p. 12], it seems that the argument above could, perhaps, have been written down a century ago.

\section{PROOF BY MiCROLOCALISATION}

To emphasize why the proofs of Proposition 1.3 and Theorem 1.1 work, the proof of the general Theorem 2.1 below will use the Fourier transformation $\mathscr{F}$ more consistently.

To apply $\mathscr{F}$ to non-integrable functions, it is convenient to use a few elements of the distribution theory of L. Schwartz [Sch66]. (An introduction to this could be [RY90].)

Recall that $\mathscr{F} f(\tau)=\hat{f}(\tau)=\int_{\mathbb{R}} e^{-\mathrm{i} t \tau} f(t) d t$ defines a bijection $\mathscr{F}: \mathscr{S}(\mathbb{R}) \rightarrow \mathscr{S}(\mathbb{R})$, when $\mathscr{S}(\mathbb{R})$ denotes the Schwartz space of rapidly decreasing $C^{\infty}$-functions. Moreover, $\mathscr{F}$ extends by duality to the space $\mathscr{S}^{\prime}(\mathbb{R})$ of so-called temperate distributions, which contains $L_{p}(\mathbb{R})$ for $1 \leq p \leq \infty$. In particular it applies to exponential functions $e^{\mathrm{i} b t}$, and as a basic exercise this 
yields $2 \pi$ times the Dirac measure $\delta_{b}$, ie the point measure at $\tau=b$,

$$
\mathscr{F}\left(e^{\mathrm{i} b \cdot}\right)(\tau)=2 \pi \delta(\tau-b)=2 \pi \delta_{b}(\tau)
$$

This applies in a discussion of the function

$$
f(t)=\sum_{j=0}^{\infty} a_{j} e^{\mathrm{i} b_{j} t}
$$

with general amplitudes $a_{j} \in \mathbb{C}$ and frequencies $0<b_{0}<b_{1}<\cdots<b_{j}<\ldots$ with $b_{j} \rightarrow \infty$, written $0<b_{j} \nearrow_{\infty}$ for brevity. (There could be finitely many $b_{j} \leq 0$, but this would only contribute with a $C^{\infty}$-term.)

Obviously the condition $\sum_{j}\left|a_{j}\right|<\infty$ implies $f \in C(\mathbb{R}) \cap L_{\infty}(\mathbb{R})$, so $f \in \mathscr{S}^{\prime}(\mathbb{R})$, and since $\mathscr{F}$ applies termwise (it is continuous on $\mathscr{S}^{\prime}(\mathbb{R})$ ), one has by (2.1)

$$
\mathscr{F} f=\sum_{j=0}^{\infty} a_{j} \mathscr{F}\left(e^{\mathrm{i} b_{j^{*}}}\right)=2 \pi \sum_{j=0}^{\infty} a_{j} \delta_{b_{j}} .
$$

Of course (2.2) just expresses that $f$ is synthesized from the frequencies $b_{0}, b_{1}, \ldots$

When $\liminf \frac{b_{j+1}}{b_{j}}>1$, then each frequency may be picked out in a well-known way: fixing $\lambda \in] 1, \liminf \frac{b_{j+1}}{b_{j}}\left[\right.$ there is a $\chi \in \mathscr{S}(\mathbb{R})$ for which $\hat{\chi}(1)=1$ while $\hat{\chi}(\tau) \neq 0$ only for $\frac{1}{\lambda}<\tau<\lambda$. Then $b_{k}>\lambda b_{k-1}$ for all $k \geq K$, if $K$ is chosen appropriately.

Considering only $k \geq K$ in the following, one has $\hat{\chi}\left(\tau / b_{k}\right) \neq 0$ only for $\left.\tau \in\right] \frac{b_{k}}{\lambda} ; \lambda b_{k}[$. Because $\left.\left[\frac{b_{k}}{\lambda} ; \lambda b_{k}\right] \subset\right] b_{k-1} ; b_{k+1}\left[\right.$ and $\left(b_{k}\right)$ is monotone increasing,

$$
\hat{\chi}\left(\tau / b_{k}\right) \delta_{b_{j}}(\tau)= \begin{cases}0 & \text { for } j \neq k \\ \delta_{b_{k}} & \text { for } j=k\end{cases}
$$

In general $\mathscr{F}(\chi * f)=\hat{\chi} \cdot \hat{f}$ holds for all $\chi \in \mathscr{S}(\mathbb{R})$ and $f \in L_{\infty} \subset \mathscr{S}^{\prime}(\mathbb{R})$, whence

$$
\mathscr{F}\left(b_{k} \chi\left(b_{k}\right) * f\right)=\hat{\chi}\left(\cdot / b_{k}\right) \cdot \mathscr{F} f=2 \pi \sum_{j=0}^{\infty} a_{j} \hat{\chi}\left(\cdot / b_{k}\right) \delta_{b_{j}}=2 \pi a_{k} \delta_{b_{k}} .
$$

So by use of $\mathscr{F}^{-1}$ and (2.1),

$$
b_{k} \chi\left(b_{k} \cdot\right) * f(t)=2 \pi a_{k} \mathscr{F}^{-1} \delta_{b_{k}}(t)=a_{k} e^{\mathrm{i} b_{k} t} .
$$

This gives back (1.8) in case $a_{k}=2^{-k \theta}$ and $b_{k}=2^{k}$, but the derivation above is more transparent than eg the proof of (1.8), since it is clear why convolution by $b_{k} \chi\left(b_{k} \cdot\right)$ just gives the $k^{\text {th }}$ term.

The process in (2.4)-(2.5) has of course been known for ages, but with distribution theory it is fully justified although $\mathscr{F} f$ consists of measures. In principle, it is a banal example of what is sometimes called a second microlocalisation of $f$, since $\hat{\chi}\left(b_{k} \tau\right) \mathscr{F} f(\tau)$ is localised to frequencies $\tau$ restricted in both size and direction; namely to $|\tau| \approx b_{k}$ and $\tau>0$, respectively. 
The second microlocalisation is more visible in a separate treatment of

$$
\operatorname{Re} f(t)=\sum_{j=0}^{\infty} a_{j} \cos \left(b_{j} t\right), \quad \operatorname{Im} f(t)=\sum_{j=0}^{\infty} a_{j} \sin \left(b_{j} t\right) .
$$

Indeed, by Euler's formula and (2.1),

$$
\mathscr{F} \cos \left(b_{j} \cdot\right)=\frac{2 \pi}{2}\left(\delta_{b_{j}}+\delta_{-b_{j}}\right), \quad \mathscr{F} \sin \left(b_{j} \cdot\right)=\frac{2 \pi}{2 \mathrm{i}}\left(\delta_{b_{j}}-\delta_{-b_{j}}\right) .
$$

Here multiplication by $\hat{\chi}\left(\cdot / b_{j}\right)$ removes the contribution from $\delta_{-b_{j}}$ since $\hat{\chi}$ vanishes on $\left.]-\infty, 0\right]$. This actually explains why the proof of Theorem 1.1 was saved by the redundancy of the term $e^{\mathrm{i} b^{j}\left(t-t_{0}\right)}$.

However, the details will follow in connection with the next result. Recall that $f: \mathbb{R} \rightarrow \mathbb{C}$ is said to be Lipschitz continuous at $t_{0}$ if there exist two constants $L>0, \eta>0$ such that $\mid f(t)-$ $f\left(t_{0}\right)|\leq L| t-t_{0} \mid$ for every $\left.t \in\right] t_{0}-\eta, t_{0}+\eta[$.

Theorem 2.1. Let $f: \mathbb{R} \rightarrow \mathbb{C}$ be given as $f(t)=\sum_{j=0}^{\infty} a_{j} \exp \left(\mathrm{i} b_{j} t\right)$ for a complex sequence $\left(a_{j}\right)_{j \in \mathbb{N}_{0}}$ with $\sum_{j=0}^{\infty}\left|a_{j}\right|<\infty$ and $0<b_{j} \nearrow \infty$ satisfying

$$
\liminf _{j \rightarrow \infty} \frac{b_{j+1}}{b_{j}}>1, \quad a_{j} b_{j} \not>0 \text { for } \quad j \rightarrow \infty .
$$

Then $f$ is bounded and continuous on $\mathbb{R}$, but nowhere differentiable. If $\sup _{j}\left|a_{j}\right| b_{j}=\infty$ holds in addition, then $f$ is not Lipschitz continuous at any point. The conclusions are also valid for $\operatorname{Re} f$ and $\operatorname{Im} f$.

Proof. Continuing from (2.5), one clearly has $a_{k} e^{\mathrm{i} b_{k} t_{0}}=\int_{\mathbb{R}} \chi(z) f\left(t_{0}-z / b_{k}\right) d z$.

If $f$ were differentiable at $t_{0}$, then $F(t)=\left(f\left(t_{0}+t\right)-f\left(t_{0}\right)\right) / t$ would be in $L_{\infty}$ (like $f$ ), so since $\int \chi(t) d t=0$, multiplication by $b_{k}$ and majorised convergence would imply

$$
-a_{k} b_{k} e^{\mathrm{i} b_{k} t_{0}}=\int_{\mathbb{R}} z \chi(z) \frac{f\left(t_{0}-z / b_{k}\right)-f\left(t_{0}\right)}{-z / b_{k}} d z \underset{k \rightarrow \infty}{\longrightarrow} f^{\prime}\left(t_{0}\right) \mathrm{i} \frac{d \hat{\chi}}{d \tau}(0)=0 .
$$

This would entail $\left|a_{k}\right| b_{k} \rightarrow 0$ for $k \rightarrow \infty$, in contradiction of (2.8).

In addition, were $f$ Lipschitz continuous at $t_{0}$, then again $F$ would be bounded, so the integral in (2.9) would be uniformly bounded with respect to $k$, in which case $\sup _{k}\left|a_{k}\right| b_{k}<\infty$.

Finally, using (2.7) $\mathrm{ff}$, one can clearly replace $f$ in (2.4)-(2.5) by $\operatorname{Re} f$ or $\operatorname{Im} f$ if only $a_{k}$ is replaced by $a_{k} / 2$ and $a_{k} /(2 \mathrm{i})$, respectively. Eg

$$
\hat{\chi}\left(\tau / b_{k}\right) \mathscr{F} \operatorname{Im} f(\tau)=2 \pi \sum_{j=0}^{\infty} \hat{\chi}\left(\tau / b_{k}\right) \frac{a_{j}}{2 \mathrm{i}}\left(\delta_{b_{j}}(\tau)-\delta_{-b_{j}}(\tau)\right)=2 \pi \frac{a_{k}}{2 \mathrm{i}} \delta_{b_{k}}(\tau)
$$

Proceeding as for $f$ itself via variants of (2.5) and (2.9), it follows that neither $\operatorname{Re} f \operatorname{nor} \operatorname{Im} f$ can be differentiable at some $t_{0} \in \mathbb{R}$, respectively Lipschitz continuous if $\sup _{j}\left|a_{j}\right| b_{j}=\infty$. 
Clearly $\lim \sup \left|a_{j}\right| b_{j}>0$ is equivalent to $a_{j} b_{j} \not \rightarrow 0$; cf (2.8). While the former leaves a gap to the non-Lipschitz condition, the latter is natural as termwise differentiation yields $\sum a_{j} b_{j} e^{i b_{j} t}$, which cannot converge unless $a_{j} b_{j} \rightarrow 0$. The conditions (2.8) have been used repeatedly in the literature, but Theorem 2.1 should be of interest because of the easy treatment of non-periodic $f$ as well as of $\operatorname{Re} f, \operatorname{Im} f$.

Remark 2.2. A necessary condition for Hölder continuity of order $\alpha \in] 0,1[$ follows at once from a modification of the above argument: replacing $a_{k} b_{k}$ on the left-hand side of (2.9) by $a_{k} b_{k}^{\alpha}$, the resulting integral will be uniformly bounded with respect to $k$ since $\int|z|^{\alpha}|\chi(z)| d z<\infty$. Hence

$$
\sup _{k}\left|a_{k}\right| b_{k}^{\alpha}<\infty
$$

whenever $f(t)$ in Theorem 2.1 is Hölder continuous of order $\alpha$ at a single point $t_{0}$.

Example 2.3. Sequences of power type like $a_{j}=a^{-j}$ and $b_{j}=b^{j}$ for parameters $b \geq|a|>1$ give $f(t)=\sum_{j=0}^{\infty} a^{-j} e^{\mathrm{i} b^{j} t}$, which is covered by Theorem 2.1 as $\left|a_{j}\right| b_{j}=\left|\frac{b}{a}\right|^{j} \geq 1$ and $\frac{b_{j+1}}{b_{j}}=b>1$. Therefore Theorem 2.1 contains Theorem 1.1 and extends it to complex amplitudes.

For $W(t) \operatorname{Remark} 2.2$ yields $\frac{b^{\alpha}}{a} \leq 1$, ie $\alpha \leq \frac{\log a}{\log b}$. In case $b>a>1$, Hardy's proof strategy [Har16, p. 311] was to show that $W$ is Hölder continuous of order $\alpha=\log a / \log b$ but no better (even locally); whereas for $b=a>1$ it was obtained that $W(t+h)-W(t)=\mathscr{O}(|h| \log 1 /|h|)$. So Remark 2.2 at once gives a sharp upper bound for the Hölder exponent of $W$. (This was mentioned as a difficult task in [Jaf97]; however, [BD92] contains a relatively short proof of the bound.) Thm. 4.9 in Ch. II of Zygmund's book [Zyg59] also treats Hölder continuity of $W$.

Example 2.4. In the same way Theorem 2.1 covers Darboux's function $f(t)=\sum_{j=0}^{\infty} \frac{\sin ((j+1) ! t)}{j !}$, for $a_{j}=1 / j$ ! and $b_{j}=(j+1)$ ! fulfil in particular $\frac{b_{j+1}}{b_{j}}=j+2 \nearrow \infty$ and $a_{j} b_{j}=j+1 \nearrow \nearrow_{\infty}$.

Example 2.5. Setting $a_{j}=a^{-j}$ for some $a>1$ and defining $\left(b_{j}\right)$ by $b_{2 m}=a^{2 m}$ and $b_{2 m+1}=$ $\left(1+a^{-p}\right) a^{2 m}$, it is seen directly that when the power $p$ is so large that $1+a^{-p}<a^{2}$, then the sequences $\left(a_{j}\right)$ and $\left(b_{j}\right)$ fulfil the conditions of Theorem 2.1. Eg (2.8) holds as $\frac{b_{j+1}}{b_{j}} \in\{1+$ $\left.\left.a^{-p}, a^{2}\left(1+a^{-p}\right)^{-1}\right\} \subset\right] 1, \infty\left[\right.$ and $a_{j} b_{j} \in\left\{1,\left(1+a^{-p}\right) / a\right\}$. Thus $f(t)$ is nowhere differentiable in this case. If further $p$ is so large that $1+a^{2}<a^{p}\left(a^{2}-1\right)$ it is easily verified that $b_{2 m+1}-b_{2 m}<$ $b_{2 m}-b_{2 m-1}$ so that $\left(b_{j+1}-b_{j}\right)$ is not monotone increasing. Eg if $a=5$, both requirements are met by $p=1$ and the values of $\left(b_{j}\right)$ are

$$
1, \frac{6}{5}, 25,30,625,750,15625,18750, \ldots
$$

Clearly these frequencies have a distribution with lacunas of rather uneven size. 
To elucidate the assumptions in Theorem 2.1, note that for a sequence $\left(b_{j}\right)$ of positive reals, $\liminf \frac{b_{j+1}}{b_{j}}>1 \Longleftrightarrow \liminf \frac{b_{j+1}-b_{j}}{b_{j}}>0 \Longleftrightarrow \exists J, \varepsilon>0 \forall j>J: \varepsilon b_{j}<b_{j+1}-b_{j}<b_{j+1}$.

Hence the conditions $\liminf \frac{b_{j+1}}{b_{j}}>1$ and $b_{j} \nearrow \infty$ imply that $b_{j+1}-b_{j} \rightarrow \infty$ when Theorem 2.1 applies; but the gaps $b_{j+1}-b_{j}$ need not be monotone increasing; cf Example 2.5.

Moreover, $\liminf \frac{b_{j+1}}{b_{j}}>1$ implies exponential growth of the $b_{j}$ (as $b_{j} \geq \lambda^{j-j_{0}} b_{j_{0}}$ when $\frac{b_{j+1}}{b_{j}} \geq$ $\lambda>1$ for $j \geq j_{0}$ ) so Thm. 2.1 does not apply if $b_{j}=j^{q}$. This will be remedied in Thm. 3.1 ff.

\section{DiLATION BY DIFFERENCES}

To escape the exponential frequency growth in Theorem 2.1 it is natural instead of dilation by $b_{j}$ to use the smallest gap at frequency $b_{j}$, ie to dilate by

$$
\Delta b_{j}=\min \left(b_{j}-b_{j-1}, b_{j+1}-b_{j}\right) . \quad\left(b_{-1}=0\right)
$$

This requires $\lim \Delta b_{j}=\infty$, that one could use as an assumption (replacing exponential growth by one of its consequences, ef (2.12). However, (3.2) below is weaker, since it only implies the existence of $j_{1}<j_{2}<\ldots$ satisfying $\lim \Delta b_{j_{k}}=\infty$ (the $\Delta b_{j}$ are unbounded since $\left.a_{j} \rightarrow 0\right)$.

Theorem 3.1. Let $f(t)=\sum_{j=0}^{\infty} a_{j} \exp \left(\mathrm{i} b_{j} t\right)$ for a complex sequence $\left(a_{j}\right)_{j \in \mathbb{N}_{0}}$ with $\sum_{j=0}^{\infty}\left|a_{j}\right|<\infty$ and $0<b_{j} \nearrow \infty$. When $\Delta b_{j}$ in (3.1) fulfils

$$
a_{j} \Delta b_{j} \not \rightarrow 0 \text { for } j \rightarrow \infty
$$

then $f$ is bounded and continuous on $\mathbb{R}$, but nowhere differentiable. If $\sup _{j}\left|a_{j}\right| \Delta b_{j}=\infty$ holds in addition, then $f$ is not Lipschitz continuous at any $t_{0} \in \mathbb{R}$. The conclusions are also valid for $\operatorname{Re} f$ and $\operatorname{Im} f$.

Proof. That $f \in C(\mathbb{R}) \cap L_{\infty}(\mathbb{R})$ is shown as in Theorem 2.1. Let now $\mathscr{F} \psi \in C^{\infty}(\mathbb{R})$ fulfil $\mathscr{F} \psi(0)=1$ and $\mathscr{F} \psi(\tau) \neq 0$ only for $|\tau|<1 / 2$, and take the spectral cut-off function as

$$
\hat{\psi}_{k}(\tau)=\hat{\psi}\left(\frac{\tau-b_{k}}{\Delta b_{k}}\right)
$$

Then the definition of $\Delta b_{k}$ as a minimum entails

$$
\hat{\psi}_{k}(\tau) \neq 0 \Longrightarrow b_{k}-\frac{1}{2}\left(b_{k}-b_{k-1}\right)<\tau<b_{k}+\frac{1}{2}\left(b_{k+1}-b_{k}\right) .
$$

Since $\left(b_{j}\right)$ is increasing, the $\tau$-interval specified here only contains $b_{j}$ for $j=k$, whence

$$
\hat{\psi}_{k}(\tau) \hat{f}(\tau)=2 \pi \sum_{j=0}^{\infty} a_{j} \hat{\psi}_{k}(\tau) \delta_{b_{j}}(\tau)=2 \pi a_{k} \delta_{b_{k}}(\tau)
$$


Note that by a change of variables,

$$
\psi_{k}(t)=\mathscr{F}^{-1} \hat{\psi}_{k}(t)=\frac{1}{2 \pi} \int_{\mathbb{R}} e^{\mathrm{i} t\left(b_{k}+\sigma \Delta b_{k}\right)} \hat{\psi}(\sigma) \Delta b_{k} d \sigma=\left(\Delta b_{k}\right) e^{\mathrm{i} t b_{k}} \psi\left(t \Delta b_{k}\right) .
$$

Here the integral of the left-hand side is 0 by (3.4), so application of $\mathscr{F}^{-1}$ to $(\underline{3.5})$ gives,

$$
\begin{aligned}
a_{k}\left(\Delta b_{k}\right) e^{\mathrm{i} b_{k} t_{0}} & =\left(\Delta b_{k}\right) f * \psi_{k}\left(t_{0}\right) \\
& =\int_{\mathbb{R}}\left(f\left(t_{0}-t\right)-f\left(t_{0}\right)\right)\left(\Delta b_{k}\right)^{2} e^{\mathrm{i} b_{k} t} \psi\left(t \Delta b_{k}\right) d t . \\
& =\int_{\mathbb{R}} \frac{f\left(t_{0}-z / \Delta b_{k}\right)-f\left(t_{0}\right)}{z / \Delta b_{k}} z \psi(z) e^{\mathrm{i} z b_{k} / \Delta b_{k}} d z .
\end{aligned}
$$

If $f$ is Lipschitz continuous at $t_{0}, h \mapsto\left(f\left(t_{0}+h\right)-f\left(t_{0}\right)\right) / h$ is bounded, so for some $L \in \mathbb{R}$

$$
\sup _{k}\left|a_{k}\right| \Delta b_{k} \leq \sup _{k} \int_{\mathbb{R}}\left|\frac{f\left(t_{0}-z / \Delta b_{k}\right)-f\left(t_{0}\right)}{z / \Delta b_{k}}\right| z \psi(z)\left|d z \leq L \int_{\mathbb{R}}\right| z \psi(z) \mid d z<\infty .
$$

Moreover, because $b_{k} / \Delta b_{k} \geq b_{k} /\left(b_{k}-b_{k-1}\right)>1$,

$$
\int_{\mathbb{R}} z \psi(z) e^{\mathrm{i} z b_{k} / \Delta b_{k}} d z=\mathrm{i} \frac{d \hat{\psi}}{d \tau}\left(-b_{k} / \Delta b_{k}\right)=0 .
$$

So were $f$ differentiable at $t_{0}$, it would follow from (3.7) by majorised convergence that

$$
a_{k}\left(\Delta b_{k}\right) e^{\mathrm{i} t_{0} b_{k}}=-\int_{\mathbb{R}}\left(\frac{f\left(t_{0}-z / \Delta b_{k}\right)-f\left(t_{0}\right)}{-z / \Delta b_{k}}-f^{\prime}\left(t_{0}\right)\right) z \psi(z) e^{\mathrm{i} z b_{k} / \Delta b_{k}} d z \underset{k \rightarrow \infty}{\longrightarrow} 0,
$$

in contradiction of (3.2). Finally the same arguments apply to $\operatorname{Re} f, \operatorname{Im} f$ by dividing $a_{k}$ by 2 and $2 \mathrm{i}$, respectively, as in Theorem 2.1 .

Remark 3.2. If $f$ in Theorem 3.1 is Hölder continuous of order $\alpha \in] 0,1\left[\right.$ at some $t_{0}$, (3.7) yields

$$
\sup _{j}\left|a_{j}\right|\left(\Delta b_{j}\right)^{\alpha}<\infty .
$$

When applied to $W$, this gives the same result as Remark 2.2, for $\Delta b_{j}=c b^{j}$ with $c=1-1 / b>0$ as $b>1$. Hence the gap growth condition (3.11) cannot be sharpened in general.

Note that, due to the use of the extended $\mathscr{F}$ on $\mathscr{S}^{\prime}(\mathbb{R})$, it is clear from (3.5) that one cannot dilate by larger quantities than $\Delta b_{k}$, so the method seems optimally exploited.

Apparently, nowhere-differentiability has not been obtained under the weak assumptions of Theorem 3.1 before. Like for $f_{\theta}$ and $W$, the regularity of the sum function improves when the growth of the frequencies is taken smaller, eg by reducing $q$ in the following:

Example 3.3 (Polynomial growth). For $p>1$ one has uniformly continuous functions

$$
f_{p, q}(t)=\sum_{j=1}^{\infty} \frac{\exp \left(\mathrm{i} t j^{q}\right)}{j^{p}}, \quad \operatorname{Re} f_{p, q}(t), \quad \operatorname{Im} f_{p, q}(t),
$$


that moreover are $C^{1}$ and bounded with bounded derivatives on $\mathbb{R}$ in case $0<q<p-1$. However, for $q \geq p+1$ they are nowhere differentiable according to Theorem 3.1: (3.2) follows since by the mean value theorem the frequency gaps increase, and

$$
\limsup j^{-p}\left(j^{q}-(j-1)^{q}\right) \geq \limsup q j^{q-p-1}(1-1 / j)^{q-1}= \begin{cases}q & \text { for } q=p+1, \\ \infty & \text { for } q>p+1 .\end{cases}
$$

Moreover, for $q>p+1$ there is not Lipschitz continuity at any point.

But the functions in (3.12) are globally Hölder continuous of order $\alpha=(p-1) / q$ if only $q>p-1$. This results from integral comparisons that (eg for $c=1+1 /(q-p+1)$ ) yield

$$
\left|f_{p, q}(t+h)-f_{p, q}(t)\right| \leq \sum_{j \leq N} j^{q-p}|h|+\sum_{j>N} 2 j^{-p} \leq c N^{q-p+1}|h|+\frac{2}{p-1} N^{1-p} .
$$

For $0<|h| \leq \frac{1}{2}$ this is exploited for the unique $N$ such that $N \leq|h|^{-1 / q}<N+1$. For the Hölder exponents, this is optimal among the powers $|h|^{-\theta}$, for clearly $\theta=1 / q$ maximises

$$
\min (\theta(p-1), 1-\theta(q-p+1)) .
$$

Insertion of the choice of $N$ in (3.14) gives a $C<\infty$ so that for $h \in \mathbb{R}$ (as $f_{p, q} \in L_{\infty}$ )

$$
\left|f_{p, q}(t+h)-f_{p, q}(t)\right| \leq C|h|^{\alpha}, \quad \alpha=\frac{p-1}{q} .
$$

Since $\Delta b_{j}<q j^{q-1}$ for $q>1$, the necessary condition in Remark 3.2 is fulfilled for $\alpha(q-1)-$ $p \leq 0$, leading to the upper bound $\alpha \leq \frac{p}{q-1}$. So in view of (3.16) there remains a gap for these functions.

In view of Example 3.3, it is clear that Theorem 3.1 improves Theorem 2.1 a good deal. The condition $\left|a_{j}\right| \Delta b_{j} \not \rightarrow 0$ in (3.2) cannot be relaxed in general, for already for $W$ it amounts to $b \geq a$, that is equivalent to nowhere-differentiability.

However, (3.2) does not give optimal results for $f_{p, q}$. Eg the case with $p=q=2$ has been completely clarified and shown to have a delicate nature, as it is known from several investigations that the so-called Riemann function

$$
R(t)=\sum_{j=1}^{\infty} \frac{\sin \left(\pi j^{2} t\right)}{j^{2}}
$$

is differentiable with $R^{\prime}(t)=-1 / 2$ exactly at $t=r / s$ for odd integers $r, s$. For properties of this function the reader is referred to the paper of J. Duistermaat [Dui91].

As $f_{p, q}$ is in $C^{1}(\mathbb{R})$ for every $q<p-1$ when $p>1$, transition to nowhere-differentiability occurs (perhaps gradually) as $q$ runs through the interval $[p-1, p+1$ [. Nowhere-differentiability for $q \geq p+1$ was also mentioned for $\operatorname{Im} f_{p, q}$ by W. Luther [Lut86] as an outcome of a very general Tauberian theorem. (In addition $\operatorname{Im} f_{p, 2}$ was covered with nowhere-differentiability for $p \leq 3 / 2$ 
providing cases in $\left[p+\frac{1}{2}, p+1[\right.$; for $t$ irrational Luther's result relied on Hardy's investigation [Har16], that covered $\operatorname{Im} f_{p, 2}$ for $p<5 / 2$ thus giving cases of almost nowhere differentiability in $\left.] p-\frac{1}{2}, p+\frac{1}{2}\right]$ for $q=2$.)

By (3.16), $R$ is globally Hölder continuous of order $\alpha=1 / 2$, that is well known [Dui91]. At the differentiability points, this is of course not optimal, but the local Hölder regularity of $R$ is known to attain every value $\alpha \in\left[\frac{1}{2}, \frac{3}{4}\right]$ in a non-empty set; cf the paper of S. Jaffard [Jaf97].

Remark 3.4. Recently $f_{p, q}$ was studied by F. Chamizo and A. Ubis [CU07] for $q \in \mathbb{N}, p>1$, with nowhere-differentiability treated by convolving $f_{p, q}$ with the Fejér kernel, cf [CU07, Prop. 3.3]. This method was proposed as an alternative to those of [Lut86] and similar in spirit to the above proof of Theorem 3.1. But some statements are flawed: eg in [CU07, Thm. 3.1], $f_{p, q}$ is claimed differentiable at an irreducible fraction $t=r / s \in \mathbb{Q}, s>0$, if and only if both $q<p+1 / 2$ and, for some maximal prime power $\sigma^{\gamma}$ in the factorisation of $s, q$ divides $\gamma-1$ but is relatively prime with $\sigma-1$. However, $f_{p, q} \in C^{1}(\mathbb{R})$ for every $q<p-1$ (cf Example 3.3 above), whilst for $q \in \mathbb{N} \cap\left[2, p-1\left[\right.\right.$ their condition is violated at $\frac{r}{s}=\frac{1}{2^{q}}$; hence this claim is not correct for such $q$.

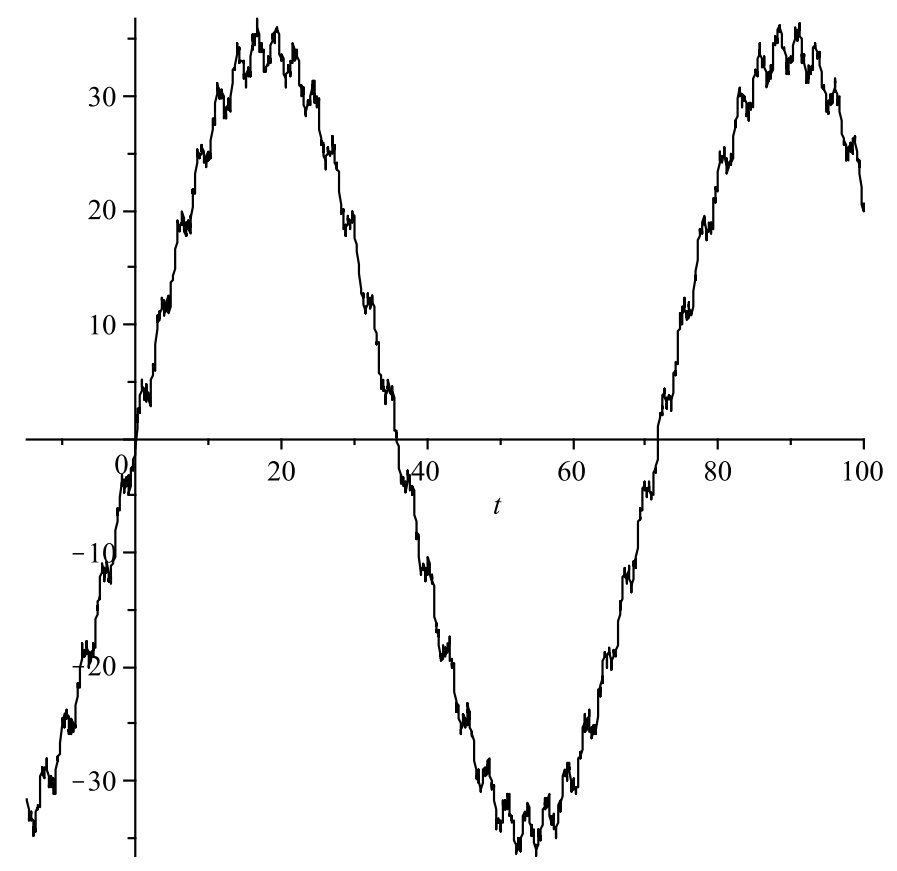

FIGURE 1. Graph of $\operatorname{Im} F_{2}(t)$ for the function in (4.3) 


\section{SLOWLY GROWING FREQUENCIES}

Using Theorem 3.1, nowhere-differentiability is obtained in new cases where $b_{j}$ is almost $\mathscr{O}\left(j^{2}\right)$. The examples to follow all relate to the limiting case $p=1, q=2$ in Example 3.3 .

Setting $\log ^{a} t=(\log t)^{a}$ for $a \in \mathbb{R}$ and $t>1$, the functions

$$
F_{1}(t)=\sum_{j=2}^{\infty} \frac{\exp \left(\mathrm{i} t j^{2} \log ^{b} j\right)}{j \log ^{a} j}, \quad \operatorname{Re} F_{1}(t), \quad \operatorname{Im} F_{1}(t)
$$

are for $b \geq a>1$ continuous, bounded and nowhere differentiable on $\mathbb{R}$ by Theorem 3.1, for the mean-value theorem gives $a_{j} \Delta b_{j} \geq 2 \log ^{b-a} j$. For $b>a>1$ there is no Lipschitz continuity.

To simplify, the Lipschitz aspect is left out below by taking $b=a$. Instead iterated logarithms will be seen to allow quasi-quadratic growth of the $b_{j}$, relying on a general result for $b_{j}=j /\left|a_{j}\right|$ :

Corollary 4.1. If $\sum_{j>J}\left|a_{j}\right|<\infty$ and $\left|a_{j}\right| \geq\left|a_{j+1}\right|>0$ for all $j>J$ while for a convex function $\varphi:] J, \infty\left[\rightarrow \mathbb{R}\right.$ one has $\varphi(j)=j /\left|a_{j}\right|$ for $\left.j \in \mathbb{N} \cap\right] J, \infty[$, then

$$
f(t)=\sum_{j>J} a_{j} \exp \left(\mathrm{i} t j /\left|a_{j}\right|\right), \quad \operatorname{Re} f(t), \quad \operatorname{Im} f(t)
$$

are continuous on $\mathbb{R}$ but nowhere differentiable.

Proof. As $\varphi$ is convex, clearly $\Delta b_{j}=\varphi(j)-\varphi(j-1)$. Therefore $\left|a_{j}\right| \Delta b_{j}=\left|a_{j}\right|\left(\frac{j}{\left|a_{j}\right|}-\frac{j-1}{\left|a_{j-1}\right|}\right) \geq$ $j-(j-1)=1$, and $b_{j}=j /\left|a_{j}\right| \nearrow \infty$, whence Theorem 3.1 yields the claim.

For $t>e$, there is a nowhere differentiable function given by

$$
F_{2}(t)=\sum_{j=3}^{\infty} \frac{\exp \left(\mathrm{i} t j^{2} \log j(\log \log j)^{a}\right)}{j \log j(\log \log j)^{a}}, \quad a>1 .
$$

This can be seen directly from Corollary 4.1, but it is a special case of Example 4.2 below.

The graph of $\operatorname{Im} F_{2}$ is sketched in Figure 1. All figures give a plot of a partial sum with 1000 terms and partition points. The quasi-periodic behaviour visible in Figure 1 results because the first term of the series is dominating. More pronounced cases of slow growth are given in:

Example 4.2. Denoting the $n$-fold $\log$ arithm by $\log _{\circ} t:=\log \ldots \log t$, defined for $t>E_{n-2}:=$ $\exp \ldots \exp 1$ ( $n-2$ times), and setting $\log _{\circ n}^{a} t=\left(\log _{\circ n} t\right)^{a}$ for $a \in \mathbb{R}$ and $t>E_{n-1}$, there is a continuous nowhere differentiable function given for $t>E_{n-1}$ by

$$
F_{n}(t)=\sum_{j>E_{n-1}} \frac{\exp \left(\mathrm{i} t j^{2} \log j \ldots \log _{\circ(n-1)} j \cdot \log _{\circ}^{a} j\right)}{j \log j \ldots \log _{\circ(n-1)} j \cdot \log _{\circ}^{a} j}, \quad a>1 .
$$

Indeed, $\sum\left|a_{j}\right|<\infty$ because $a_{j}=1 /\left(j \log j \ldots \log _{\circ(n-1)} j \log _{\circ n}^{a} j\right)$ equals $g^{\prime}(j)$, whereby

$$
g(t)=\frac{1}{1-a} \log _{\circ n}^{1-a} t=\frac{1}{1-a}(\log \ldots \log t)^{1-a} \underset{t \rightarrow \infty}{\longrightarrow} 0 \quad \text { for } \quad a>1 .
$$


That $a_{j} \geq a_{j+1}$ follows since all iterated logarithms are monotone increasing and positive for $j>E_{n-1}$. Analogously, $\varphi_{a, n}(t)=t^{2} \log t \ldots \log _{\circ(n-1)} t \cdot \log _{\circ n}^{a} t$ is convex on $] E_{n-1}, \infty\left[\right.$, for $\varphi_{a, n}^{\prime}(t)$ is easily written as a sum of $n+1$ terms, that are increasing. Hence Corollary 4.1 gives the claim.

$\operatorname{Im} F_{3}$ and its first term are sketched in Figure 2 for $a=2$. One has $E_{2}=e^{e} \approx 15.15$, and for $j \geq 16$ the frequencies are $0.28,1.4,3.5,6.8,11,17,25,34,45,57, \ldots$ (As $a=2$, the sum could include $e<j<e^{e}$, but the $b_{j}$ decrease from 11 for $j=6$ to 0.009 for $j=15$.)

As a last comparison, for $a=2$ and $n=4$, summation begins in (4.4) after $E_{3}=e^{e^{e}}=$ $3814279.1 \ldots$ Cf Figure 3. The quasi-quadratic growth of $b_{j}$ is indicated by the fact that terms no. $1,10,100,1000$ have frequencies $0.02,2.4,247,24326$, respectively.

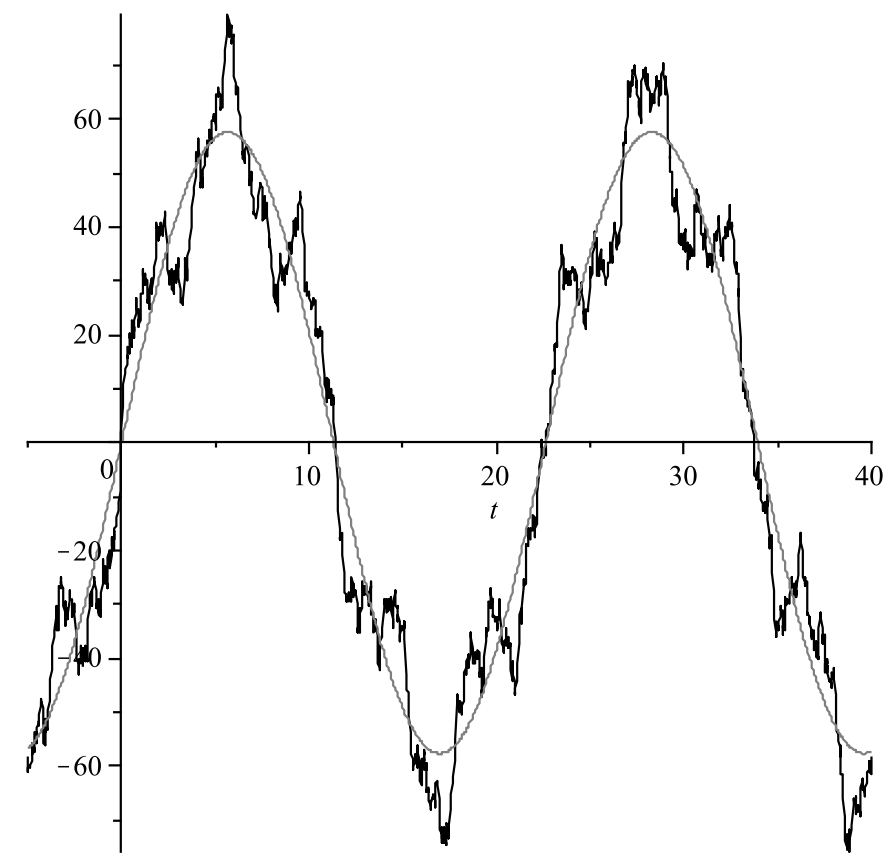

FIGURE 2. $\operatorname{Im} F_{3}(t)$ for the function in Example 4.2, $n=3, a=2$

It may of course be shown analytically that, despite the larger number of $j$-dependent factors, one gets slower frequency growth in $F_{n+p}$ than in $F_{n}$. Figures 1, 2 and 3 indicate that as the frequency growth is reduced, there will be increasingly larger deviations from a sinusoidal curve.

Figure 4 shows the deviation from the first term, ie the sum over $j \geq 3814281$. Notice that here the sinusoidal structure is almost completely lost, ie the first term is even less dominating.

In addition to the vertical tangent at the origin in Figure 4, there seems to be approximate self-similarities, like those for $R$ analysed by J. Duistermaat [Dui91]. Eg the behaviour for ca. $40<t<75$ seems similar to that found for $25<t<40$ and so on for $t \rightarrow 0_{+}$. 


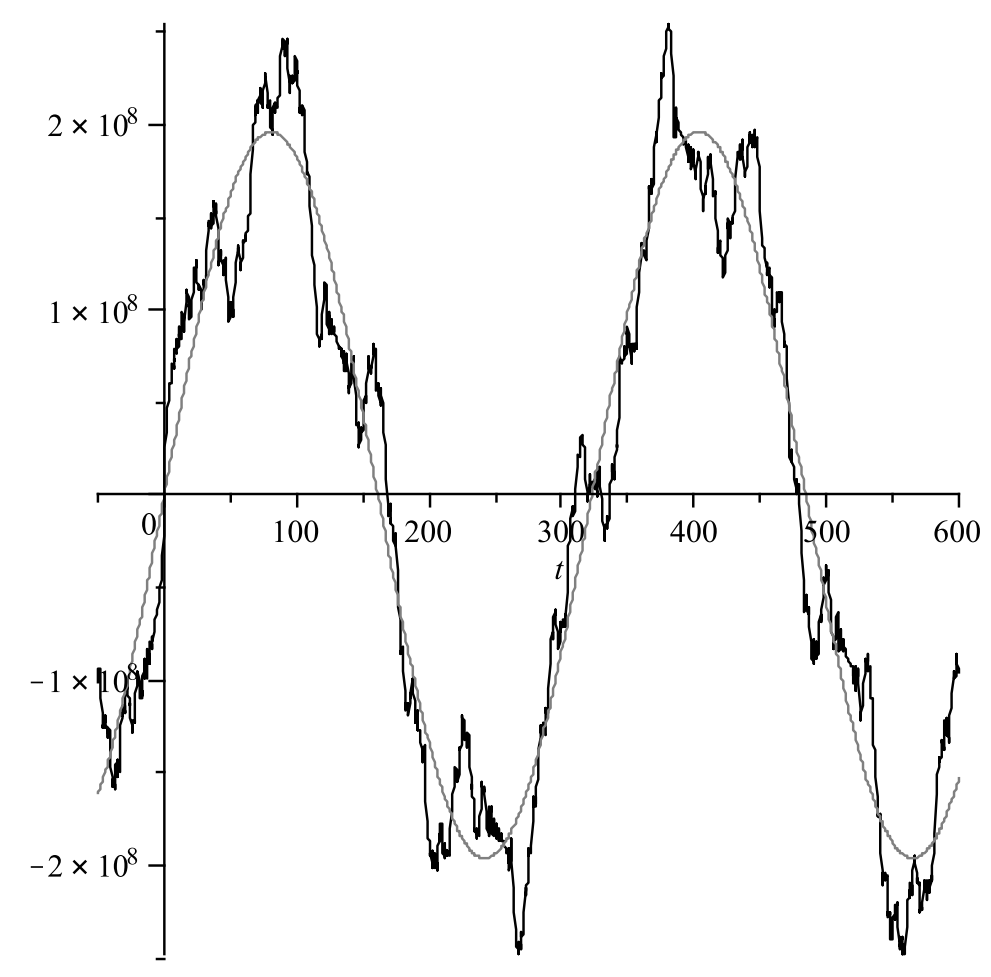

FIGURE 3. $\operatorname{Im} F_{4}(t)$ for the function in Example 4.2, $n=4, a=2$

\section{FINAL REMARKS}

The first example of a nowhere differentiable function is due to B. Bolzano (ca. 1830, discovered 1921), cf the accounts in [Hyk01, Thi03]. Nowhere-differentiability was established by means of infinite products in [Wen02]. For a review of the historical development of the subject the reader could consult the illustrated thesis of J. Thim [Thi03]. Very recently nowheredifferentiable functions were shown to enter the counter-examples that establish the pathological properties of pseudo-differential operators of type 1, 1; cf [Joh08].

It has turned out that some elements of the arguments exist sporadically in the literature; cf Remark 3.4 for comments on [CU07]. In particular $\operatorname{Re} f_{\theta}$ and $\operatorname{Im} f_{\theta}, \theta=1$ have been analysed by Y. Meyer [Mey93, Ch. 9.2] with a method partly based on wavelets and partly similar to the proof of Proposition 1.3. The method was attributed to G. Freud but without any references.

However, G. Freud showed in [Fre62] that an integrable periodic function $f$ with Fourier series $\sum \rho_{k} \sin \left(n_{k} t+\varphi_{k}\right), \inf n_{k+1} / n_{k}>1$ is differentiable at a point only if $\lim \rho_{k} n_{k}=0$, similarly to Theorem 2.1. His proof was based on estimates of the differentiated Cesaro means and of the corresponding Fejér kernel (as done also in [SS03]), so it applies only to periodic functions. 


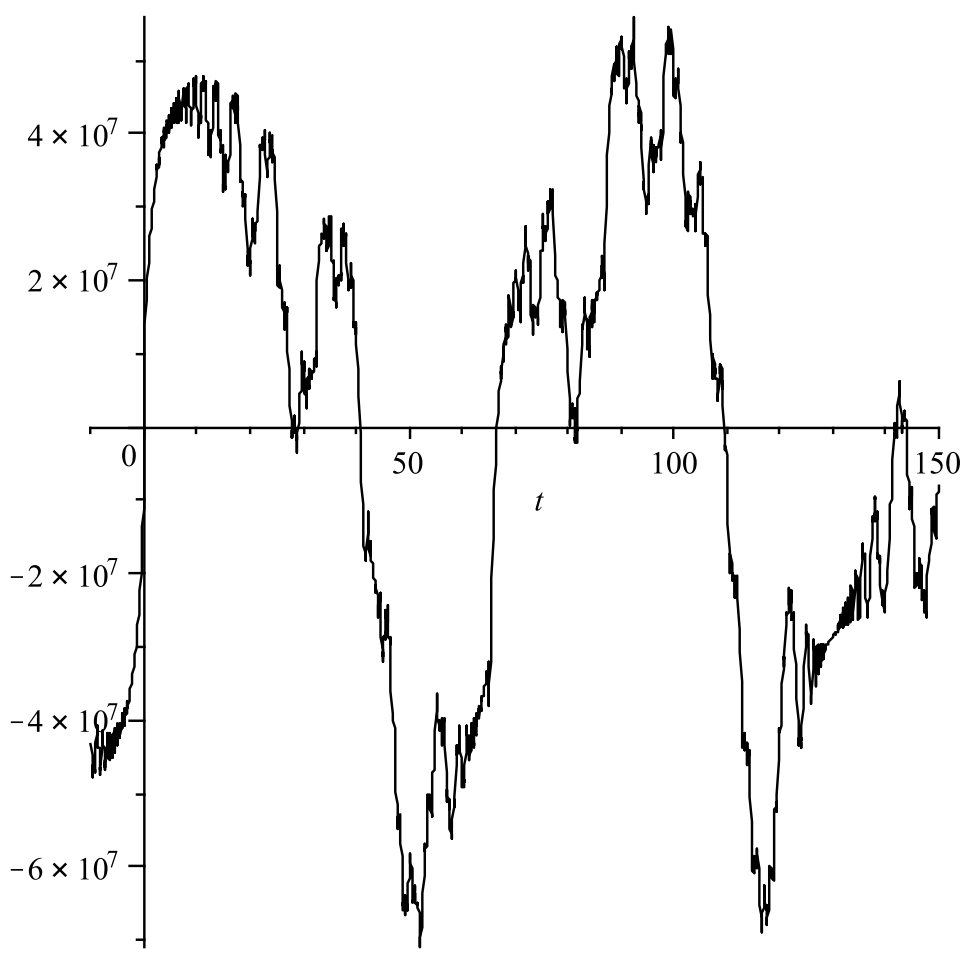

FIGURE 4. Deviation from the first term of $\operatorname{Im} F_{4}(t)$ in Example 4.2, $n=4, a=2$

Whereas the purpose in [Mey93, Ch. 9.2] was to derive the lack of differentiability of $\operatorname{Re} f_{1}$, $\operatorname{Im} f_{1}$ with wavelet theory, the present paper goes much beyond this. Eg nowhere-differentiability of $f_{\theta}$, or $W$, is shown to follow directly from basic facts in integration theory; cf the introduction. And using only $\mathscr{F}$, differentiability was in Theorem 2.1 linked to the growth of the frequencies $b_{j}$. Finally, the removal of the condition $\liminf b_{j+1} / b_{j}>1$ in Theorem 3.1 seems to be a novelty, which yields that the growth of the frequency increments $\Delta b_{j}$ is equally important.

Acknowledgement. I am grateful to Professor L. Rodino and Professor H. Cornean for asking me to publish this work; and to an anonymous referee for pointing out the reference [BD92].

\section{REFERENCES}

[BD92] A. Baouche and S. Dubuc, La non-dérivabilité de la fonction de Weierstrass, L'Enseignement de Math. 38 (1992), 89-94.

[CU07] F. Chamizo and A. Ubis, Some Fourier series with gaps, J. Anal. Math. 101 (2007), 179-197.

[Dui91] J. J. Duistermaat, Self-similarity of “Riemann's nondifferentiable function”, Nieuw Arch. Wisk. (4) 9 (1991), no. 3, 303-337.

[Fre62] G. Freud, Über trigonometrische Approximation und Fouriersche Reihen, Math. Z. 78 (1962), $252-262$.

[Har16] G. H. Hardy, Weierstrass's non-differentiable function, Trans. Amer. Math. Soc. 17 (1916), no. 3, 301325. 
[Hol95] M. Holschneider, Wavelets, Oxford Mathematical Monographs, The Clarendon Press Oxford University Press, New York, 1995, An analysis tool, Oxford Science Publications.

[Hyk01] M. Hykšová, Bolzano's inheritance research in Bohemia, Mathematics throughout the ages (Holbæk, 1999/Brno, 2000), Děj. Mat./Hist. Math., vol. 17, Prometheus, Prague, 2001, pp. 67-91.

[Jaf97] S. Jaffard, Old friends revisited: the multifractal nature of some classical functions, J. Fourier Anal. Appl. 3 (1997), 1-22.

[Joh08] J. Johnsen, Type 1,1-operators defined by vanishing frequency modulation, New Developments in PseudoDifferential Operators (L. Rodino and M. W. Wong, eds.), Operator Theory: Advances and Applications, vol. 189, Birkhäuser, 2008, pp. 201-246.

[Leb08] H. Lebesgue, Sur la méthode de M. Goursat pour la résolution de l'équation de Fredholm, Bull. Soc. Math. France 36 (1908), 3-19.

[Lut86] W. Luther, The differentiability of Fourier gap series and "Riemann's example" of a continuous, nondifferentiable function, J. Approx. Theory 48 (1986), 303-321.

[McC53] J. McCarthy, An everywhere continuous nowhere differentiable function, Amer. Math. Monthly 60 (1953), 709.

[Mey93] Y. Meyer, Wavelets, Society for Industrial and Applied Mathematics (SIAM), Philadelphia, PA, 1993, Algorithms \& applications.

[RY90] I. Richards and H. Youn, Theory of distributions. A nontechnical introduction, Cambridge University Press, Cambridge, 1990.

[Sch66] L. Schwartz, Théorie des distributions, revised and enlarged ed., Hermann, Paris, 1966.

[SS03] E. M. Stein and R. Shakarchi, Fourier analysis, Princeton Lectures in Analysis, vol. 1, Princeton University Press, Princeton, NJ, 2003.

[Tak03] T. Takagi, A simple example of the continous function without derivative, Phys.-Math. Soc. Japan 1 (1903), 176-177, The Collected Papers of Teiji Takagi, S. Kuroda, Ed.,Iwanami (1973), 5-6.

[Thi03] J. Thim, Continuous nowhere differentiable functions, Master's thesis, Luleå University of Technology, Sweden, 2003.

[Wen02] Liu Wen, A nowhere differentiable continuous function constructed by infinite products, Amer. Math. Monthly 109 (2002), 378-380.

[Zyg59] A. Zygmund, Trigonometric series. 2nd ed. Vols. I, II, Cambridge University Press, New York, 1959.

Department of Mathematical Sciences, Aalborg University, Fredrik Bajers vej 7G, DK9220 AALBORG ØST, DENMARK

E-mail address: jjohnsen@math.aau.dk 